\title{
Article \\ Determinants of Reactive Agility in Tests with Different Demands on Sensory and Motor Components in Handball Players
}

\author{
Henrieta Horníková ${ }^{1, *(D)}$, Michal Jeleň ${ }^{2}\left(\mathbb{D}\right.$ and Erika Zemková ${ }^{1,2}(\mathbb{D}$ \\ 1 Department of Biological and Medical Sciences, Faculty of Physical Education and Sport, \\ Comenius University, 81469 Bratislava, Slovakia; erika.zemkova@uniba.sk \\ 2 Faculty of Electrical Engineering and Information Technology, Sports Technology Institute, \\ Slovak University of Technology, 81219 Bratislava, Slovakia; michal.jelen@stuba.sk \\ * Correspondence: hornikova.henrieta@gmail.com; Tel.: +421-915-301-044
}

Citation: Horníková, H.; Jeleň, M.;

Zemková, E. Determinants of

Reactive Agility in Tests with Different Demands on Sensory and Motor Components in Handball Players. Appl. Sci. 2021, 11, 6531. https://doi.org/10.3390/app11146531

Academic Editor: Herbert Wagner

Received: 29 May 2021

Accepted: 13 July 2021

Published: 15 July 2021

Publisher's Note: MDPI stays neutral with regard to jurisdictional claims in published maps and institutional affiliations.

Copyright: (c) 2021 by the authors. Licensee MDPI, Basel, Switzerland. This article is an open access article distributed under the terms and conditions of the Creative Commons Attribution (CC BY) license (https:// creativecommons.org/licenses/by/ $4.0 /)$.

\begin{abstract}
This study investigates the relationship between reactive agility and reaction speed, sprint speed, and muscle strength and their contribution to Y-shaped agility test and reactive agility test (RAT) performance in handball players. Seven handball players performed a Y-shaped agility test and RAT, simple and choice-based reaction time test, reaction test to fast and slow generated visual stimuli, $5 \mathrm{~m}$ and $20 \mathrm{~m}$ sprint tests, a 505 Agility test, a squat jump, a countermovement jump, and a drop jump. The results reveal a significant relationship between the times for the Y-shaped agility test and the $20 \mathrm{~m}$ sprint $(\mathrm{r}=0.777, p=0.040, \mathrm{R}=0.604)$, as well as the time for the 505 Agility test $\left(\mathrm{r}=0.770, p=0.043, \mathrm{R}^{2}=0.593\right)$, and RSI $\left(\mathrm{r}=-0.755, p=0.050, \mathrm{R}^{2}=0.570\right)$; however, no relationship was found with parameters pertaining to reaction speed. RAT performance did not significantly correlate with RSI, time in the 505 Agility test, squat jump height, time in the $20 \mathrm{~m}$ sprint, and reaction time to fast generated visual stimuli, although large effect sizes were found $(r>0.5)$. It seems that the contribution of sensory and motor components depends on the structure of the agility test. While the Y-shaped agility test is mainly determined by sprinting, the change of direction speed, and reactive strength, there is the tendency for the reactive agility test to be determined by strength, speed, and visual reaction time.
\end{abstract}

Keywords: explosive strength; change of direction speed; reaction speed; reactive agility test; reactive strength; sprint speed; Y-shaped agility test

\section{Introduction}

Agility is an important skill for both attackers and defenders in invasion sports [1]. It is generally accepted that agility has both movement and reactive elements, whereas predetermined change of direction (COD) tasks without a requirement to react to a stimulus are described by COD ability [2]. Some authors use the term "reactive agility" to make a clear distinction from agility including only change of direction speed (CODS) $[3,4]$. It is necessary to deconstruct this skill into smaller and more manageable components to better understand agility. The first deterministic model in this context was published in 2002 [5], which indicated that agility includes two subcomponents, i.e., the CODS component and perception and decision-making component, which are determined by many other factors. The assessment of reactive agility is more complex because of the requirement to react to a stimulus in contrast with only pre-planned COD movements. As a result, some researchers have used a flashing light device to direct athletes to new locations [6-9].

Researchers have identified reactive agility as one of the most important determinants of performance in team sports $[3,6,10,11]$. Handball, as a team sport, involves many multidirectional changes of direction that occur mostly in response to unpredictable stimuli (e.g., the ball or an opponent, etc.) in a 40-m-long and 20-m-wide court [12]. Reactive 
agility is assessed by both stop-and-go and non-stop running scenarios in handball $[9,13]$. Similar to COD assessments, there are a few key characteristics that can be used to compare results. The first is the time to complete the test and the second is the number of directional changes the test requires, as well as the degrees of these direction changes [2]. For example, a change in direction in the range of $0^{\circ}$ to $90^{\circ}$ is velocity-oriented, whereas a change in direction in the range of $90^{\circ}$ to $180^{\circ}$ is force-oriented [14]. In addition, reactive agility tests including a perception and decision-making component may differ in terms of the number of possible responses. For instance, a reaction to one stimulus may be recorded as $279 \mathrm{~ms}$, with the response to two stimuli as $511 \mathrm{~ms}$ and four stimuli as $804 \mathrm{~ms}$. Notably, a reaction time of $\sim 0.5 \mathrm{~s}$ (reaction time to two stimuli) may be important in everyday situations, as well as in sports [15].

Although the structures of reactive agility components and their subcomponents in invasion sports are well known, there is no evidence as to what extent perceptual and decision-making processes and motor components contribute to reactive agility in a different sport types. Most of these studies concern only soccer/football, whereas, for example, handball or tennis have been investigated to a lesser extent. From a practical point of view, it would be interesting to know whether the contribution of sensory and motor components depends on the given structure of reactive agility testing. The most used reactive agility tests include Y-shaped agility testing and sport-specific reactive agility testing. As such, the first test considered in this study was a Y-shaped agility test, which is considered valid and reliable for measuring reactive agility performance [8]. This test included forward running $(5 \mathrm{~m})$ and reaction to one visual stimulus which then determined a directional change to the right or left in a $45^{\circ}$ angle that was then followed by another sprint for $5 \mathrm{~m}$. The second test, which considered a reactive agility test (RAT) modified from Sekulic et al. [10], included 20 movement reactions to 4 visual stimuli placed in a semicircle at a distance of $2 \mathrm{~m}$. This indicates that a Y-shaped agility test can be considered as a velocity-oriented test with non-stop running, whereas a RAT is a force-oriented test rather than a velocity-oriented stop-and-go test. This is because repeated short distance CODS sprints in response to visual stimuli require a great amount of explosive movement; however, the question remains regarding the extents to which speed and strength variables are associated with reactive agility how they contribute to performance. In this study, we test the hypothesis that Y-shaped agility test performance is mainly determined by motor factors, whereas RAT performance is primarily determined by sensory factors rather than motor-based factors. Verification of this hypothesis was accomplished by investigation of the relationship between reactive agility and reaction speed, sprint and change of direction speed, explosive and reactive strength of lower limbs and their contribution to Y-shaped agility test and RAT in handball players.

\section{Materials and Methods}

\subsection{Participants}

Seven competitive male handball players participated in this study (20.9 \pm 0.5 years, $183.3 \pm 4.9 \mathrm{~cm}, 85.6 \pm 8.1 \mathrm{~kg}$ ). A requirement for selection in the study was to be an active handball player. All of the participants were professional players of the first or second highest league in the Slovak Republic and had $10.6 \pm 2.1$ years of experience in a particular sport. They trained an average six times a week and regularly played matches. Goalkeepers did not participate in this study. All participants were informed of the procedures and the main purpose of the study. The procedures presented here were conducted in accordance with the ethical standards for human experimentation as stated in the Helsinki Declaration.

\subsection{Experimental Procedure}

A standard warm-up routine was performed prior to the study, including light aerobic warm-up and dynamic stretching exercises. The participants then attended a familiarization session where the testing conditions were explained and trial sets were carried out. Firstly, participants performed reaction time tests using the FiTRO Reaction Check 
(FiTRONiC, Bratislava, Slovakia) and Witty SEM (Microgate, Bolzano, Italy) diagnostic systems. Secondly, jumping was measured by the OptoGait (Microgate, Bolzano, Italy) system and sprint tests, change of direction speed tests, and reactive agility tests were measured by the Witty SEM (Microgate, Bolzano, Italy) system.

\section{Y-Shaped Agility Test}

Y-shaped agility testing has previously been found to be reliable and valid [8]. A Witty light-based timing system was used to record times and set reactive conditions. The gates were set at a width of $1.5 \mathrm{~m}$ and a height of $1.2 \mathrm{~m}$. A goniometer was used to determine the $45^{\circ}$ angle of the change of direction. The test consisted of a 5-m-long sprint, a $45^{\circ}$ change in direction to the left or right, and finally a 5-m-long sprint to the finish gate. The timing system dictated the direction in which participants proceeded after having completed the first $5 \mathrm{~m}$ of the course. The photocell of the timing system presented an arrow denoting a change in direction to the right or left with an approximate 40 to $45 \mathrm{~ms}$ delay after passing by the starting gate. Trials were performed in triplicate and the best times were used for analysis.

Reactive Agility Test (RAT)

The reactive agility test that was used was a modified version of the stop-and-go reactive agility test developed by Sekulic et al. [10]. In this test, four photocells were placed $2 \mathrm{~m}$ from the starting point in a semi-circular arrangement. The task for the participant was to run to the lit photocell as fast as possible, wave at this photocell, and then run back to the starting point. The test consisted of 20 visual reactions (five in each direction) with a generation time of $1 \mathrm{~s}$. A green light was used as a stimulus, while the other photocells remained off. The result of the test was a total time for one trial.

\section{Simple and Choice Reaction Time Test}

The FiTRO Reaction Check diagnostic system consists of two switches connected by means of an interface to a computer. Special software measured the time between the stimulus appearing and the participant's response. Participants were required to respond to either one visual stimulus (a simple reaction time test) or two visual stimuli in the form of a circle and cross (a two-choice reaction time test) positioned on switches on a table. The switches had to be pressed in accordance with the stimulus on the screen. Participants had to press the right-most switch when a green circle appeared and the left-most switch when a red cross appeared. Participants performed 2 trials of 20 responses in each test. The data from the best trials of simple reaction time and 2-choice reaction time testing were selected for analysis.

\section{Reaction Time Test to Fast Generating Visual Stimuli}

The Witty SEM diagnostic system used in this study included four photocells which were situated in-line on a table with a distance of $10 \mathrm{~cm}$ between them. Participants were required to react as fast as possible with their dominant hand to photocells which lit up green. Both tests consisted of 20 visual reactions, i.e., 4 for each photocell. In this test, the visual stimuli were generated immediately after each response. The final result was the best total reaction time after two trials.

Squat Jump (SJ)

A squat jump is a jump without previous countermovement that starts from a $90^{\circ}$ knee position (measured by a goniometer) with the hands on the hips. Participants were instructed to jump as high as possible from a semi-squatting position without countermovement. The final result of the test was the highest jump of two trials.

\section{Countermovement Jump (CMJ)}

A CMJ is performed from a standing position, where participants bent their knees approximately 90 degrees and then immediately jumped as high as possible. The participants performed two jumps and the higher of them was recorded for statistical analysis. 


\section{Drop Jump (DJ)}

For the drop jump test, participants started from an upright position on a 30-cm-high box while keeping their hands placed on their hips. Participants were instructed to drop down from the box between two bars of the OptoGait system and rebound with a very quick ground contact time and minimal knee bending. The participants had to land with both feet at the same time and not bend their knees or kick their feet when landing. The result of this jump was a reactive strength index (RSI) that was calculated by the software as the ratio of the drop jump height and the time contact in the best of two trials.

\section{$20 \mathrm{~m}$ and $5 \mathrm{~m}$ Sprint}

A 20-m-long sprint with $5 \mathrm{~m}$ split time were used as an assessment of linear sprint speed. Witty timing gates were positioned at $0 \mathrm{~m}, 5 \mathrm{~m}$, and $20 \mathrm{~m}$. Gates were set at a width of $1.5 \mathrm{~m}$ and height of $1.2 \mathrm{~m}$. Participants started sprinting $30 \mathrm{~cm}$ behind the start line to trigger the first gate and were instructed to sprint maximally through each gate. The time results of the $5 \mathrm{~m}$ split and $20 \mathrm{~m}$ sprint in the better of two trials were analysed.

\section{Agility Test}

CODS was assessed with a 505 Agility test. All participants performed one trial on each leg with a 2-min rest between trials. Participants started the test $0.3 \mathrm{~m}$ behind the photocell gates to prevent early triggering of the initial start gate. The timing gates were again placed at the hip height. Participants were instructed to sprint to a line marked $15 \mathrm{~m}$ away from the start line, place either their left or right foot on the line, depending on the trial, then turn $180^{\circ}$ and sprint back over a length of $5 \mathrm{~m}$ through the finish time gate. The better performance of two trials was used for further analysis.

\subsection{Statistical Analyses}

All statistical analyses were computed using the statistical program SPSS for Windows (version 22.0, SPSS, Inc., Chicago, IL, USA). Shapiro-Wilk testing was performed for all variables and revealed that the data obtained were normally distributed. Pearson correlation coefficients were used to find the relationships between the reactive agility performance and reaction, sprint speed, and muscle strength parameters. A correlation coefficient (r) less than 0.30 was considered small, 0.31 to 0.49 moderate, and 0.50 and above as a large effect [16]. Additionally, coefficients of determination $\left(R^{2}\right)$ were used to estimate the contributions of measured variables to reactive agility. The level for statistical significance was set at $p<0.05$. Descriptive statistics (mean $\pm \mathrm{SD}$ ) were calculated for all results. The statistical power analysis and effect sizes were calculated using $G^{*}$ power 3.1 for Windows. An a priori sample size estimate for an alpha level of 0.05 , effect size of 0.5 , and power (1-ß probability error) of 0.80 was calculated. A total sample size of 26 participants appeared to be necessary to detect the relationship between reactive agility tests and the reaction, speed, and strength parameters; however, the post hoc sample size estimate for an alpha level of 0.05 and effect size of 0.71 (calculated from $R^{2}=0.50$ ) dropped the power to 0.57 . The sample size in this study was below the limit because the inclusion criteria required participants to be active in handball and train regularly, which made participation difficult due to COVID-19 restrictions at the time.

\section{Results}

The results of the tests described in this study are shown in Table 1.

The time in Y-shaped agility test significantly correlated with the time in the 20-m-long sprint, time in the 505 Agility test, and RSI in the drop jump test $(r=0.777, r=0.770$, and $\mathrm{r}=-0.755$, respectively). A negative correlation means that a higher RSI resulted in a shorter agility time in the Y-shaped agility test. All correlation coefficients are shown in Table 2. 
Table 1. Descriptive statistics of handball player test performance.

\begin{tabular}{cc}
\hline Test & Mean \pm SD \\
\hline Simple reaction time & $315 \pm 19.6$ \\
\hline Choice reaction time & $409 \pm 18.9$ \\
\hline Reaction time to fast generated visual stimuli & $10.3 \pm 0.5$ \\
\hline Time in the Y-shaped agility test & $2.0 \pm 0.1$ \\
\hline Time in the reactive agility test (RAT) & $40.2 \pm 2.3$ \\
\hline Squat jump height & $29.6 \pm 4.1$ \\
\hline Countermovement jump height & $34.9 \pm 5.4$ \\
\hline RSI in a drop jump test & $1.5 \pm 0.4$ \\
\hline
\end{tabular}

Table 2. Relationship between Y-shaped agility test performance and the sprint speed and strength parameters.

\begin{tabular}{cccc}
\hline Y-Shaped Agility Test & $\mathbf{R}$ & $p$ & $\mathbf{R}^{\mathbf{2}}$ \\
\hline Simple reaction time & 0.125 & 0.789 & 0.016 \\
\hline Choice reaction time & 0.107 & 0.819 & 0.011 \\
\hline Reaction time to fast generating visual stimuli & 0.062 & 0.895 & 0.004 \\
\hline Time in 5 m sprint & 0.665 & 0.103 & 0.442 \\
\hline Time in 20 m sprint & 0.777 & 0.040 & 0.604 \\
\hline Time in the 505 Agility test & 0.770 & 0.043 & 0.593 \\
\hline Squat jump height & -0.608 & 0.148 & 0.370 \\
\hline Countermovement jump height & -0.540 & 0.210 & 0.292 \\
\hline RSI in the drop jump test & -0.755 & 0.0499 & 0.570 \\
\hline
\end{tabular}

Further analysis was provided using the parameters which significantly correlated with the time in Y-shaped agility test. The values for the 20-m-long sprint time, time for the 505 Agility test, and RSI in the drop jump test significantly correlated with each other $(\mathrm{r}=0.813, \mathrm{r}=-0.837$, and $\mathrm{r}=-0.906$, respectively). Furthermore, the time in the 20-m-long sprint significantly correlated with time in the 5-m-long sprint and squat jump height $(\mathrm{r}=0.914$ and $\mathrm{r}=-0.809$, respectively) tests. Time in the 505 Agility test significantly correlated with both time in the 5-m-long and 20-m-long sprint ( $\mathrm{r}=0.813$ and $\mathrm{r}=0.787$, respectively) tests, while the RSI in the drop jump test significantly correlated with the squat jump height $(\mathrm{r}=0.844)$ (Table 3$)$.

Table 3. Relationships between sprint and strength parameters in handball players.

\begin{tabular}{|c|c|c|c|c|c|}
\hline & 505 Agility Test & Sprint $(20 \mathrm{~m})$ & Sprint (5 m) & $\begin{array}{l}\text { RSI in the Drop } \\
\text { Jump Test }\end{array}$ & Squat Jump Height \\
\hline \multicolumn{6}{|l|}{505 Agility test } \\
\hline Sprint $(20 \mathrm{~m})$ & $0.813^{*}$ & & & & \\
\hline Sprint (5 m) & 0.787 * & $0.914^{* *}$ & & & \\
\hline $\begin{array}{l}\text { RSI in the drop } \\
\text { jump test }\end{array}$ & $-0.906^{* *}$ & $-0.837^{*}$ & -0.679 & & \\
\hline Squat jump height & -0.714 & $-0.809 *$ & -0.605 & $0.844^{*}$ & \\
\hline
\end{tabular}


Based on these findings, the hierarchical structure of the first- and second-order factors determining the Y-shaped agility test performance in handball players was determined (Figure 1).

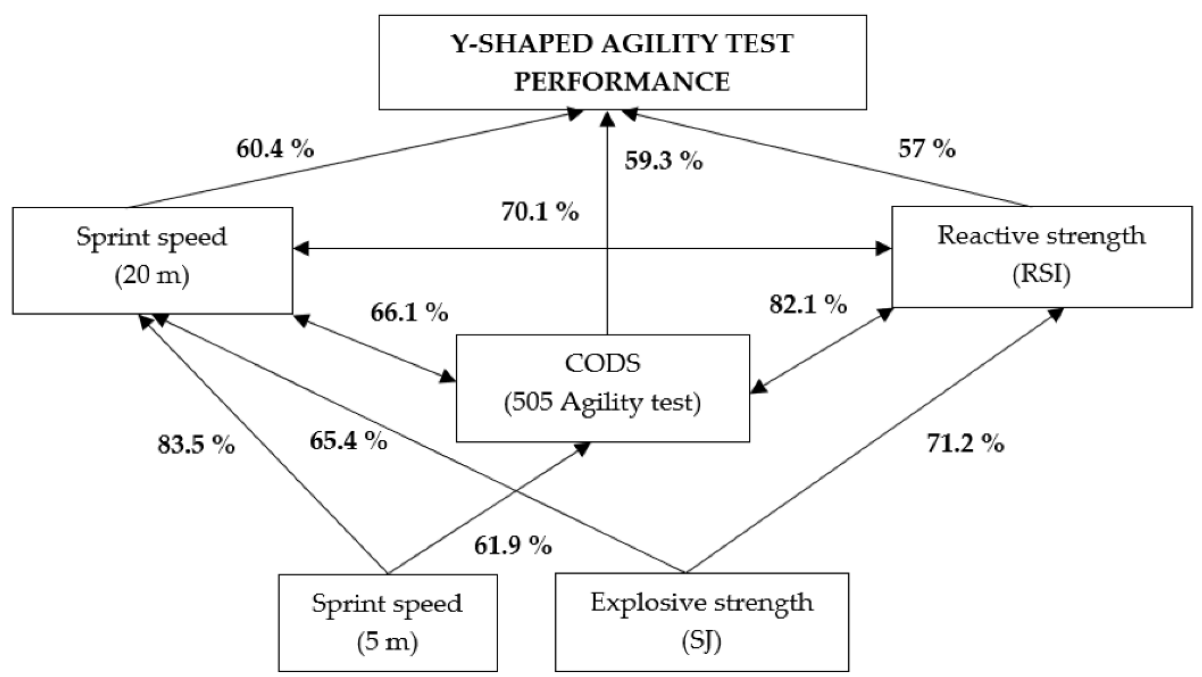

Figure 1. Percentual contributions of factors determining Y-shaped agility test performance based on the coefficients of determination for the tests described in this work for handball players.

The time in the RAT did not significantly correlate with any of the measured reaction speed, sprint speed, or muscle strength parameters; however, the relationship between the time in the RAT, RSI in the drop jump test, time in the 505 Agility test, squat jump height, time in the 20-m-long sprint, and reaction time to visual stimuli indicated large effect sizes $(r>0.5)$ (Table 4).

Table 4. Relationship between RAT performance and the reaction speed, sprint speed, and strength parameters.

\begin{tabular}{cccc}
\hline RAT & $\mathbf{r}$ & $p$ & $\mathbf{R}^{\mathbf{2}}$ \\
\hline Simple reaction time & 0.439 & 0.324 & 0.193 \\
\hline Choice reaction time & 0.220 & 0.635 & 0.048 \\
\hline $\begin{array}{c}\text { Reaction time to fast generating } \\
\text { visual stimuli }\end{array}$ & 0.486 & 0.269 & 0.236 \\
\hline Time in the 5-m-long sprint & 0.341 & 0.454 & 0.116 \\
\hline Time in the 20-m-long sprint & 0.566 & 0.185 & 0.320 \\
\hline Time in the 505 Agility test & 0.654 & 0.111 & 0.428 \\
\hline Squat jump height & -0.572 & 0.180 & 0.327 \\
\hline Countermovement ump height & -0.221 & 0.634 & 0.049 \\
\hline RSI in the drop jump test & -0.747 & 0.054 & 0.558 \\
\hline
\end{tabular}

Parameters with large effect sizes were included for further analysis. Reactive strength, change in direction speed, explosive strength, and reaction speed to visual stimuli were selected as the variables for the first-order factors (Figure 2). The second-order factors included variables that showed a significant relationship with them, which were the same as mentioned previously in Table 3 . 


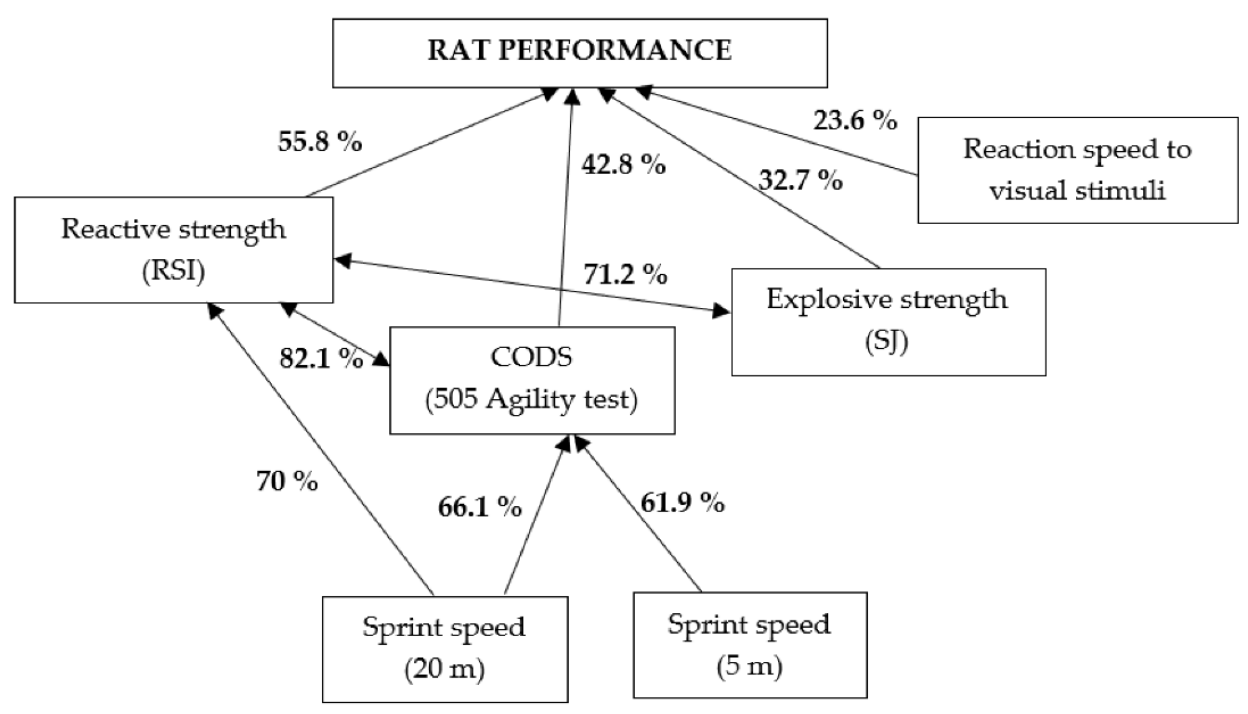

Figure 2. Percentual contribution of factors that determined the RAT performance based on the coefficients of determination for handball players.

\section{Discussion}

Performance in the Y-shaped agility test was significantly correlated with the 20-m-long sprint time, time in the 505 Agility test, and RSI in the drop jump test for the handball players considered in this work. These results are partially in agreement with a previous study which revealed a significant relationship between Y-shaped agility performance and CODS test results, but not with 10-m-, 20-m-, and 30-m-long sprint times [13]. This can be ascribed by the structure of the Y-shaped agility test, which consists of a sprint of $5+5 \mathrm{~m}$ with a change in direction of $45^{\circ}$ as per one visual stimulus, which may be classified as a velocity-oriented test with non-stop running. This type of test is characterised by lower speed loss, a shorter contact time with the ground, and a smaller number of breaking phases [14]. It is obvious that the motor component is more dominant than the sensory component in this test. Motor factors such as the CODS are important for sport performance as well as talent identification in handball [17]. As such, these results confirmed the assumption about the relationship between the Y-shaped agility test and speed factors.

In addition, there was a significant relationship between the Y-shaped agility test results and RSI results. The strength parameter is associated with the sprint and change of direction speeds, which is where a short contact time is needed [18-20]. The Y-shaped agility test also includes running with a change in direction as reaction to a visual stimulus. This may explain its significant relationship with the strength parameter.

With regards to the hierarchical model of the Y-shaped agility test, its performance is determined by the sprint speed for a longer distance, change in direction speed, and reactive strength in the first-order factors in handball players. Sprint speed for a shorter distance and explosive strength in the squat jump test determined performance in Y-shaped agility test for the second-order factors.

The RAT performance showed a large but non-significant correlation with the RSI, time in 505 Agility test, squat jump height, time in the 20-m-long sprint, and reaction time to visual stimuli in handball players. This non-significant relationship can be explained by the low sample size in this study. The significant relationship between the reactive agility time and change in direction speed $[9,21]$ and the change of direction speed and drop jump height has been found in studies with a higher number of participants [22]. In contrast to the velocity-oriented Y-shaped agility test, the RAT may be considered to be a force-oriented test. Participants in this stop-and-go test had to repeatedly accelerate and decelerate while moving over short distances in response to visual stimuli. In this type of testing, explosive strength plays an important role and may be corroborated by the large effect size of the RSI with the squat jump height. Furthermore, in the case of the 505 Agility 
test large effect size was observed. This is most likely due to the similar structure of this test, based on stop-and-go running, as in the RAT.

With regards to the hierarchical model of the RAT, its performance in the first-order factors is determined by reactive strength $(55.8 \%)$, change in direction speed $(42.8 \%)$, explosive strength $(32.7 \%)$, and reaction speed $(23.6 \%)$ in handball players. The importance of jumping capacity and reactive strength for stop-and-go CODS performance in athletes has also been proven by another study [23]. The speeds for the 5-m-long and 20-m-long sprints determined the performance in the RAT for the second-order factors due to the significant relationship with time in the 505 Agility test.

The results of this study show that the Y-shaped agility test results were mainly determined by the sprinting speed and change in direction speed, while the RAT results were primarily determined by reactive strength and the change in direction speed. These findings indicate that the motor component contributes to a higher extent to the reactive agility in handball players. It may be attributed to the long-term adaptation to sport-specific stimuli of handball players. Linear sprinting, sprinting with a change in direction with or without reaction to visual stimulus, and jumping are the most common activities in handball; however, perceptual and decision-making processes also play essential roles in determining the performance of handball players. Both sensory and motor components are very important in terms of performance in handball. In addition to motor abilities, they should focus their training programs on both sensory-motor skills. This need is also indicated by the study of Young et al. [1], in which the authors called for a focus on the coupling of perception and action in invasion sports.

The non-significant relationships between reaction speed, sprint speed, and muscular strength in the RAT was most likely due to the small sample size which was affected by the COVID-19 restrictions at the time. Further limitations should be considered in terms of using of pharmacological agents (e.g., caffeine), which was not controlled in handball players and could influence the speed of responses to visual stimuli.

\section{Conclusions}

In this study, there was a significant relationship between the time results of the Y-shaped agility testing and the 20-m-long sprint time, and 505 Agility test time, and RSI. The performance in this test was determined by the linear sprint speed $(60.4 \%)$, change in direction speed $(59.3 \%)$, and reactive strength $(57 \%)$.

Although a significant relationship between the time in the RAT and reaction speed, sprint speed, and muscle power parameters was not observed, large effect sizes indicate that the RAT performance may be determined by the reactive strength $(58.1 \%)$ and change in direction speed $(42.8 \%)$ parameters and less by explosive strength $(32.7 \%)$, and reaction speed (23.6\%). The second-order factors included sprint speed for in sprints of $20 \mathrm{~m}$ and $5 \mathrm{~m}$.

These findings indicate that the motor component contributed to performance in the Y-shaped agility test here, whereas, in the reactive agility test, the motor and sensory components contributed to performance. Consequently, speed and muscular strength can be considered as critical factors of reactive agility in handball players. As perceptual and decision-making processes also play an important role in reactive agility, it is necessary to pay more attention to the improvement of their cognitive skills. Coaches should use more exercises including running with changes of direction in reaction to visual stimuli rather than pre-planned COD movements alone.

Author Contributions: Conceptualization, H.H. and E.Z.; methodology, H.H. and E.Z.; validation, H.H. and E.Z.; formal analysis, H.H.; investigation, H.H. and M.J.; data curation, H.H. and M.J.; writing—original draft preparation, H.H., E.Z.; writing—review and editing, H.H. and E.Z.; visualization, H.H.; supervision, E.Z.; funding acquisition, E.Z. All authors have read and agreed to the published version of the manuscript.

Funding: This research was funded by the Scientific Grant Agency of the Ministry of Education, Science, Research and Sport of the Slovak Republic and the Slovak Academy of Sciences (No. $1 / 0089 / 20)$. 
Institutional Review Board Statement: The study was conducted according to the guidelines of the Declaration of Helsinki and approved by the Ethics Committee of the Faculty of Physical Education and Sports, Comenius University in Bratislava (No. 1/2020).

Informed Consent Statement: Informed consent was obtained from all subjects involved in the study.

Data Availability Statement: The data presented in this study are available on request from the corresponding author. The data are not publicly available due to ethical and privacy restrictions.

Conflicts of Interest: The authors declare no conflict of interest.

\section{References}

1. Young, W.B.; Rayner, R.; Talpey, S. It's time to change direction on agility research: A call to action. Sports Med. Open 2021, 7. [CrossRef]

2. Brughelli, M.; Cronin, J.; Levin, G.; Chaouachi, A. Understanding change of direction ability in sport a review of resistance training studies. Sports Med. 2008, 38, 1045-1063. [CrossRef]

3. Scanlan, A.; Huphries, B.; Tucker, P.S.; Dalbo, V. The influence of physical and cognitive factors on reactive agility performance in men basketball players. J. Sports Sci. 2014, 32, 367-374. [CrossRef]

4. Sheppard, J.M.; Young, W.B.; Doyle, T.L.A.; Sheppard, T.A.; Newton, R.U. An evaluation of a new test of reactive agility and its relationship to sprint speed and change of direction speed. J. Sci. Med. Sport 2006, 9, 342-349. [CrossRef] [PubMed]

5. Young, W.B.; James, R.; Montgomery, I. Is muscle power related to running speed with changes of direction? J. Sports Med. Phys. Fit. 2002, 42, 282-288.

6. Lockie, R.G.; Jeffriess, M.D.; McGann, T.S.; Callaghan, S.J.; Schultz, A.B. Planned and reactive agility performance in semiprofessional and amateur basketball players. Int. J. Sports Physiol. Perform. 2014, 9, 766-771. [CrossRef]

7. Matlák, J.; Tihanyi, J.; Racz, L. Relationship between reactive agility and change of direction speed in amateur soccer players. J. Strength Cond. Res. 2016, 30, 1547-1552. [CrossRef]

8. Oliver, J.L.; Meyers, R.W. Reliability and generality of measures of acceleration, planned agility and reactive agility. Int. J. Sports Physiol. Perform. 2009, 4, 345-354. [CrossRef] [PubMed]

9. Spasic, M.; Krolo, A.; Zenic, N.; Delextrat, A.; Sekulic, D. Reactive agility performance in handball; development and evaluation of a sport-specific measurement protocol. J. Sports Sci. Med. 2015, 14, 501-506. [PubMed]

10. Sekulic, D.; Krolo, A.; Spasic, M.; Uljevic, O.; Peric, M. The development of a new stop'n'go reactive-agility test. J. Strength Cond. Res. 2014, 28, 3306-3312. [CrossRef]

11. Young, W.; Rogers, N. Effects of small-sided game and change-of-direction training on reactive agility and change-of-direction speed. J. Sports Sci. 2014, 32, 307-314. [CrossRef]

12. Karcher, C.; Buchheit, M. On-court demands of elite handball, with special reference to playing positions. Sports Med. 2014, 44, 797-814. [CrossRef]

13. Bayraktar, I. The influences of speed, COD speed and balance on reactive agility performance in team handball. Int. J. Environ. Sci. Educ. 2017, 3, 451-461. [CrossRef]

14. Bourgeois, F.A.; McGuigan, M.R.; Gill, N.D.; Gamble, P. Physical characteristics and performance in change of direction tasks: A brief review and training considerations. J. Aust. Strength Cond. 2017, 25, 104-117.

15. Zemková, E.; Hamar, D. Towards and Understanding of Agility Performance, 2nd ed.; Albert: Boskovice, Czech Republic, 2015; ISBN 978-80-7326-258-7.

16. Cohen, J. Statistical Power Analysis for Behavioral Science, 2nd ed.; Erlbaum: Hillsdale, NJ, USA, 1988; ISBN 0-8058-0283-5.

17. Ziv, G.; Lidor, R. Physical characteristics, physiological attributes, and on-court performances of handball players: A review. Eur. J. Sport Sci. 2009, 9, 375-386. [CrossRef]

18. Castillo-Rodríguez, A.; Fernández-García, J.C.; Chinchilla-minguet, J.L.; Carnero, E.Á. Relationship between muscular strength and sprints with changes of direction. J. Strength Cond. Res. 2012, 26, 725-732. [CrossRef]

19. Flanagan, E.P.; Comyns, T. The use of contact time and the reactive strength index to optimize fast stretch-shortening cycle training. Strength Cond. J. 2008, 30, 32-38. [CrossRef]

20. Young, W.B.; Dawson, B.; Henry, G. Agility and change-of-direction speed are independent skills: Implications for agility in invasion sports. Int. J. Sports Sci. Coach. 2015, 10, 159-169. [CrossRef]

21. Šimonek, J.; Horička, P.; Hianik, J. The differences in acceleration, maximal speed and agility between soccer, basketball, volleyball and handball players. J. Hum. Sport Exerc. 2017, 12, 73-82. [CrossRef]

22. Salaj, S.; Markovic, G. Specificity of jumping, sprinting, and quick change-of-direction motor abilities. J. Strength Cond. Res. 2011, 25, 1249-1255. [CrossRef]

23. Sattler, T.; Sekulic, D.; Spasic, M.; Peric, M.; Krolo, A.; Uljevic, O.; Kondrič, M. Analysis of the association between motor and anthropometric variables with change of direction speed and reactive agility performance. J. Hum. Kinetic 2015, 47, 137-145. [CrossRef] [PubMed] 